\title{
The effect of CS-US contingency variation on GSR and on subjective CS-US relational awareness
}

\author{
KARL SCHIFFMANN and JOHN J. FUREDY \\ University of Toronto, Toronto, Ontario, Canada M5S 1 A1
}

\begin{abstract}
Ninety subjects were tested in a single-cue Pavlovian conditioning paradigm involving three groups which differed with regard to the contingency relationship between CS and US (negative contingency, zero contingency, positive contingency). To examine the relationship between conditioned GSR performance and subject's cognitive beliefs regarding the CS-US relationship, both GSR and CS-US relational learning were constantly measured on a continuous scale over the entire experimental session. The data suggest the independence of autonomic and cognitive responding in the single-cue Pavlovian paradigm.
\end{abstract}

In experimental studies of human Pavlovian conditioning, subjects are typically exposed to pairings of a conditional stimulus (CS) and an unconditional stimulus (US). As a result of this learning, subjects may exhibit at least two types of learning (Dawson, Note 1): (1) relational learning $(R L)$, whereby there is developed an awareness of the contingent relationship between the CS and the US, and (2) classical conditioning, (CC), whereby the $\mathrm{CS}$ acquires control over the US-elicited response.

The development of $\mathrm{CC}$ in the Pavlovian paradigm has been extensively investigated over the last 50 years, but the concurrent phenomenon of RL and the nature of the relationship between the two phenomena has only recently come under direct examination. The bulk of recent human conditioning research has been concerned with the cognitive issue of whether RL is an essential precondition of $\mathrm{CC}$. The weight of evidence at present suggests that autonomic Pavlovian conditioning is only found in subjects who exhibit CS-US contingency awareness (Baer \& Fuhrer, 1968, 1970; Dawson, 1970, Note 1; Dawson \& Biferno, 1973; Dawson \& Grings, 1968; Morgenson \& Martin, 1968; Shean, 1968a, b). However, possibly due to the relatively imprecise and discontinuous nature of most current measures of $R L$, the more complex question of the nature of the functional relationship between $\mathrm{RL}$ and $\mathrm{CC}$ has not been extensively examined.

The present study sought to answer two aspects of the RL-CC relationship issue. First, a continuously scaled measure of RL was employed with a single-cue Pavlovian conditioning paradigm to explore in detail the changes and development of subjects' CS-US relational awareness over the conditioning session. Second, since three experimental conditions with differing objective CS-US relations were used (to allow certain

This research, a short version of which was reported at the meeting of the Canadian Psychological Association, June 1972, was supported by grants from the National Research Council of Canada (APA 222) and from the Canada Council (S70-710) to J. J. F. We are indebted to R. J. Heslegrave for advice on an earlier draft of this paper. validation tests of the $R L$ measure), we also took the opportunity to examine the methodological implications of Rescorla's (1967, 1969b) position that CS-US contingency relations are directly related to the extent of Pavlovian conditioning performance.

\section{METHOD}

Subjects

The 90 subjects in the study were from an undergraduate subject pool at the University of Toronto and each participated for a $\$ 1.50$ payment.

\section{Apparatus}

The GSR measuring apparatus was identical to that described in Furedy and Schiffmann (1971). The instrument on which the subject was to register his subjective contingency beliefs (SC) was mounted on a small platform on the right arm of the subject's chair, and served as the measure of RL. This consisted of a lever which was spring-loaded at a vertical position but was movable $90 \mathrm{deg}$ to the left or to the right. The semicircular panel over which the lever could be moved was labeled "Scale indicating subjects' belief about whether a shock will occur in the next few moments." The vertical position was marked "uncertain," while the positions $90 \mathrm{deg}$ to the left and to the right were marked, respectively, "certainty" and "zero probability." Subjects were informed that the scale represented a continuum of SC belief ranging from certainty that shock was about to come to certainty that shock was not about to come. In other respects, the general apparatus was as specified in previous reports (Furedy \& Schiffman, 1971).

\section{Procedure}

The experiment used a single-cue conditioning arrangement with a $3,500-\mathrm{Hz}$ tone as $\mathrm{CS}$ and a $2.5-\mathrm{mA}$ electric shock as US; $\mathrm{CS}$ and US durations, respectively, were 5 and $.3 \mathrm{sec}$.

After the subject was seated and galvanic skin response (GSR) electrodes were attached, the instructions were read over the intercom system. It was emphasized that the SC scale should be used continuously throughout the experiment to register all the subtle changes in the subject's moment-to-moment beliefs about the likelihood that the shock was about to occur.

All subjects were presented with a preliminary stage comprising a CS, US, CS, US series, with the stimuli being separated by randomly chosen intervals of 30,40 , or 50 sec. During the 20-min conditioning stage, where the CS-US temporal relationship was manipulated between groups, 15 CSs and 15 USs were presented, with US occurrence being randomized over the total conditioning session. The three groups, to each of which 30 
subjects were randomly allocated, had random but identical US arrangements, and varied only in terms of the contingency relationships between the CS and US. In the CS + US group the US always immediately followed the CS offset, in a standard 5 -sec delay paradigm. The $\mathrm{zCS}$ group, which was designed to provide zero correlation between CS and US, contained $15 \mathrm{CSs}$ which were randomly distributed over the conditioning session independently of US occurrence, using a randomization technique where the 20 -min conditioning session was considered to contain $2,400.5-\sec$ time units. In the nCS group, designed to provide a negative correlation between CS and US, the CSs were placed with the constraint that the US never follow the CS within $29 \mathrm{sec}$.

\section{RESULTS}

The SC measure was sampled at 1-sec intervals from CS onset to $10 \mathrm{sec}$ after CS onset and expressed in percent full-scale units, so that fully negative, zero, and fully positive contingency beliefs were represented, respectively, by the values of $-100,0$, and +100 . From inspection of Figure 1, the SC measure appears to be fully developed at 3 and $4 \mathrm{sec}$. This method of measuring the SC response (i.e., mean of SC values at 3 and $4 \mathrm{sec}$ following CS onset) gave maximal information about SC changes over the CS-US interval. In deciding what aspect of the whole SC response best reflected the subjects' CS-US contingency beliefs, consideration was given to the fact that subjects were instructed to indicate what they "believe to be the probability of getting a shock at various moments throughout the experiment." In these terms, a tonic or absolute response measure at some particular point in the CS-US interval seemed most appropriate as reflecting the perceived SC relationship between stimuli occurring at that moment (e.g., the CS) and the US.

The GSR was defined as the resistance difference between the point of response initiation and the minimal resistance reached during the 1- to 5-sec CS-US interval. These resistance changes were transformed into micromho conductance-change units.

Responses to the preliminary stimulus series for both the SC and GSR showed no significant difference between the three groups. The SC data showed highly reliable CS-US contingency discrimination between the three conditions. For the SC response measure (for means see Figure 1), separate analyses of the significant three-level groups effect $[F(2,87)=73.09, \mathrm{MSe}=9,179.8$, $\mathrm{p}<.001]$ yielded significant $(\mathrm{p}<.01)$ differences both between the CS + US and zCS groups and between the

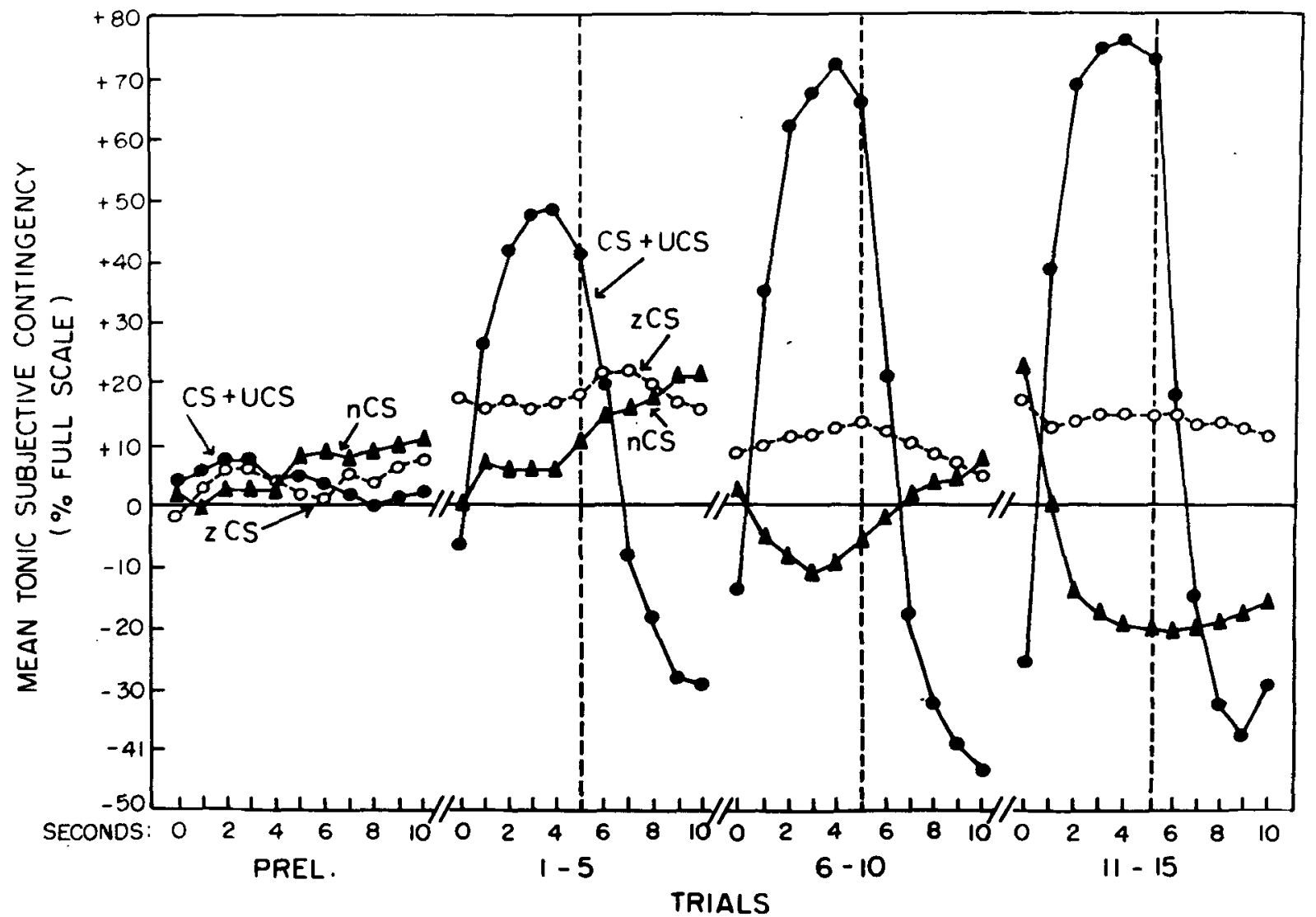

Figure 1. Mean SC responding at $1-\sec$ intervals from CS onset to $10 \mathrm{sec}$ after $\mathrm{CS}$ onset for the $\mathrm{CS}+\mathrm{US}(\mathrm{N}=30)$, $\mathrm{zCS}$ $(\mathrm{N}=30)$, and nCS $(\mathrm{N}=30)$ groups during preliminary and three blocks of conditioning trials. (The vertical interrupted lines mark the point of US onset in the CS + US group.) 
$\mathrm{zCS}$ and nCS groups.

Inspection of Figure 1, which shows SC measurements at, during, and after the CS over the course of conditioning trials, also yielded a number of other features of subject performance which can only be obtained with the sort of concurrent measurement of cognitive processes used in the present experiment. First, for the CS + US group, where the US always occurred $5 \mathrm{sec}$ after CS onset (marked with a vertical line in Figure 1), there was a clear and rapid SC decrease at US occurrence. Second, it will be noted that the measurement of the SC response at the moment of CS onset in all groups (see the zero-interval or zero-second response in Figure 1) can be considered an estimate of SC responding during the intertrial interval (ITI). As suggested by inspection of Figure 1, the pattern of these ITI SC levels varied between the three groups in both their overall mean value and their development over conditioning trials. Specifically, for the CS + US group, the mean overall ITI SC level was significantly negative $[t(29)=2.14, p<.05]$ and did not change significantly over the three conditioning trial blocks $[F(2,58)=2.69$, $\mathrm{MSe}=4,262.9, \mathrm{p}>.05]$. For the $\mathrm{zCS}$ group, mean ITI $\mathrm{SC}$ level was significantly positive $[\mathrm{t}(29)=3.69, \mathrm{p}<.01]$, but again did not change significantly over blocks $[\mathrm{F}(2,58)=2.07, \mathrm{MSe}=2,528.4, \mathrm{p}>.05]$. For the $\mathrm{nCS}$ group, the mean ITI SC level was significantly positive $[\mathrm{t}(29)=2.21, \mathrm{p}<.05]$ and increased significantly over blocks $[F(2,58)=7.74, \mathrm{MSe}=2,776.6, \mathrm{p}<.01]$, from 0.00 to 23.03. Similar differential response patterns between the three groups can also be observed from Figure 1 by comparison of the zero-interval SC responding, indicating an estimate of the ITI SC, with the tonic SC response measure, based on the mean of Intervals 3 and 4 during CS presentation. In the CS + US group, this change was significantly positive in direction $[t(29)=91.4, p<.001]$ and increased significantly over blocks $[F(2,58)=14.05, \mathrm{MSe}=2,689.6, p<.001]$. In the $\mathrm{nCS}$ group, the change was significantly negative in direction $[\mathrm{t}(29)=3.86, \mathrm{p}<.001]$ and increased significantly over blocks $[\mathrm{F}(2,58)=14.60, \mathrm{MSe}=1,832.4$, $\mathrm{p}<.001]$. In the $\mathrm{zCS}$ condition, there was no overall change in either direction during the CS, relative to the ITI SC response. Notably, the pattern of results in the zCS group conforms closely to Rescorla's (1969b, p. 65) physical specification of a "truly random" series as one where "the probability of a US is the same given either the presence or absence of the CS."

Mean GSR performance in each of the three groups is shown in Figure 2. The GSR data provide evidence for conditioning in the CS + US group. This is demonstrated by comparison with the other two groups, normally thought of as control groups (for sensitization and pseudoconditioning effects). The performance of the CS + US group significantly exceeded both the nCS group performance $[\mathrm{F}(1,58)=5.13, \mathrm{MSe}=6.66, \mathrm{p}<.05]$ and the zCS group performance $[\mathrm{F}(1,58)=6.35, \mathrm{MSe}=$

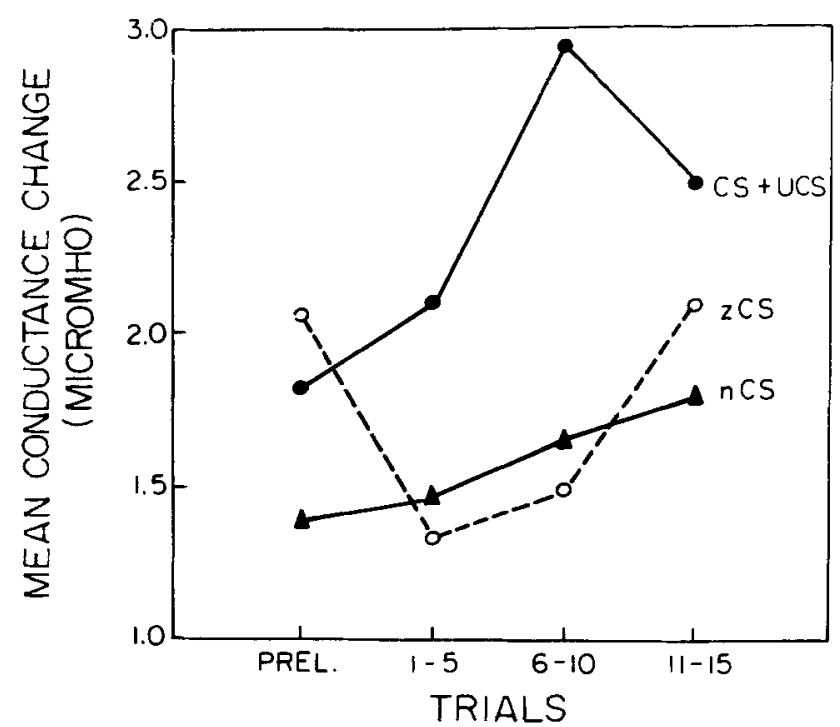

Figure 2. Mean GSR to the CS of the CS + US, zCS, and the nCS groups.

$5.50, \mathrm{p}<.05$ ]. However, in contrast to the SC data, the $\mathrm{nCS}$ and $\mathrm{zCS}$ conditions did not significantly differ $(\mathrm{F}<1)$.

The results indicate that the subjects were well able to distinguish the contingency differences between the $\mathrm{nCS}$ and $\mathrm{zCS}$ conditions (see Figure 1), but there was no analogous GSR discrimination (see Figure 2), although evidence for reliable conditioning was obtained for the CS + US group. To further explore this lack of correspondence between the electrodermal GSR and cognitive SC dependent variables, Pearson product-moment correlations between GSR and SC performance were calculated for each of the three conditions. Two forms of correlation were calculated. In the first, the trial-by-trial correlation exploited the fact that SC was measured throughout the experimental session and trial-by-trial SC data were available. These correlations were calculated from the 15 GSR and SC scores of each subject for the $15 \mathrm{CS}$ trials given during the conditioning session. In this way, each of the 30 subjects in each condition contributed one correlation coefficient. The mean for each group of these correlation coefficients was then subjected to a t-test analysis to see if each group differed significantly from zero. In the second form of correlational analysis, a mean GSR and SC score was derived for each subject so that each of the 30 subjects, in each group, contributed a pair of scores to the resulting correlation coefficient. This may be considered a less sensitive method than the trial-by-trial analysis, since this method does not include the variability over trials reported above.

The pattern of correlations, for the three conditions, from both the trial-by-trial and overall computations were as follows: The mean trial-by-trial GSR-SC correla- 
tions for both the CS + US $\left(\overline{\mathrm{X}}_{\mathrm{r}}=.06\right)$ and $\mathrm{zCS}\left(\overline{\mathrm{X}}_{\mathrm{r}}=.06\right)$ groups did not differ significantly from zero $[\mathrm{t}(29)=1.07$, $\mathrm{p}>.1$ and $\mathrm{t}(29)=1.18, \mathrm{p}>.1$, respectively], while the nCS correlation gave a significant but negative mean GSR-SC correlation $\left(\overline{\mathrm{X}}_{\mathrm{r}}=-.11\right)$ [t $\left.(29)=2.8, \mathrm{p}<.01\right]$. The SC overall correlations were also not significantly different from zero for the $\mathrm{zCS}(\mathrm{r}=.19)$ and $\mathrm{nCS}$ $(\mathrm{r}=.08)$ conditions, but for the CS + US condition $(\mathrm{r}=.33)$ the correlation was positive and significant at the .05 level.

\section{DISCUSSION}

The highly simplified single-cue conditioning paradigm employed in the present study allowed relatively easy estimation of the differing objective CS-US relations in the three experimental groups, which, in turn, facilitated assessment of the validity and sensitivity of the SC measure of RL. In terms of such sensitivity, the SC measure clearly detected differences not only between CSs paired (CS+) and not paired (zCS and nCS) with the US, but also between CS stimuli which were merely uncorrelated with $(\mathrm{zCS})$, in contrast to being negatively correlated with (nCS), the US. In this respect, the present concurrent SC measure detected no more than had the previously used, postexperimental SC measures (e.g., Schiffmann \& Furedy, 1972). However, the concurrent and continuously monitored SC measure used in this experiment also indicated other aspects of RL during the conditioning experiment. First, in addition to the orderly learning effect over trials (see, e.g., increase of CS+:nCS discrimination over trials in Figure 1), the most striking SC phenomenon was the rapid SC decrease in the CS + US condition at CS+ offset (see Figure 1), and immediately after US presentation when, consistent with objective reality, subjects estimated US occurrence probability to be at its lowest. Second, SC responding during the ITI where the CS is absent again indicated the basic accuracy of the subject's perception of the CS-US relations (see Figure 1). In the CS + US group, ITI SC responding was negative, consistent with the fact that in this condition all USs were predicted by the CS+. In the nCS condition, the ITI SC became, over trial blocks, appropriately positive, with the ITI (CS absence) being rated as more related to US occurrence than during the nCS (CS presence); during and immediately after the nCS, the SC became, over trial blocks, appropriately negative. Finally, in the $\mathrm{zCS}$ condition, which was explicitly arranged to conform with Rescorla's description of a "truly random" CS-US condition where "the probability of a US is the same given either the presence or absence of the CS" (Rescorla, 1969b, p. 65), there was indeed no difference between ITI SC responding and $\mathrm{SC}$ responding during the CS (see Figure 1).

It appears, then, that the experimental operations of uncorrelating the CS and US in the $\mathrm{zCS}$ condition were correctly perceived by subjects as indicated by the concurrent, and continuously monitored, SC measure.

This partial list of cognitive effects detected by the concurrent SC measure attests to the value of this instrument in the investigation of cognitive processes during human Pavlovian autonomic conditioning. More importantly, it provides evidence for the "construct validity" (Cronbach, 1960) of the SC measure in demonstrating the capacity of the measure to detect differences in cognitive CS-US relational beliefs when these cognitive differences might reasonably be expected to have occurred on the basis of the known experimental operations performed.

The apparent validity and sensitivity of the SC measure of RL invites an examination of the relationship between RL and autonomic performance. One of the most prominent effects was that the three experimental conditions, which were varied between subjects in terms of the relationship between the CS and US, were very reliably discriminated by the cognitive SC measure. Even though reliable between-groups autonomic conditioning was demonstrated (performance of the CS + US group exceeding that of the $\mathrm{zCS}$ and nCS groups), there was no autonomic discrimination between the $\mathrm{zCS}$ and nCS groups. That is, subjects who were apparently sensitive to, or aware of, the differeng CS-US relations of two stimuli (zCS and nCS) did not translate this relational awareness knowledge into differential autonomic behavior.

This aspect of the results could conceivably be accounted for by asserting that the GSR, in contrast to $\mathrm{SC}$, is not sensitive to the extremely fine contingency difference between $\mathrm{nCS}$ and $\mathrm{zCS}$. However, another aspect of the results which is not open to this type of differential-sensitivity argument is the correlational analysis, which also failed to reveal a consistently positive relationship between autonomic and cognitive performance. Of the six sets of coefficients calculated for the three groups for both the trial-by-trial and overall computations, one was significantly positive (as predicted from the cognitive interpretation), but one was significantly negative, and the absolute values of all the coefficients were quite low. This pattern of results is consistent with the findings of the earlier Toronto experiments, where SC was less sensitively measured (Furedy \& Schiffmann, 1971; Schiffman \& Furedy, 1972), and provides no clear support for any position which holds that the extent of autonomic Pavlovian conditioning is directly related to the extent of CS-US relational awareness.

Apart from our primary concern with documenting the course of RL during Pavlovian conditioning, the present experimental paradigm also allowed a test of an important methodological issue. Rescorla $(1967,1969 a)$ has presented evidence and arguments that CS-US contingency relations are critical determinants of Pavlovian conditioning. From such a contingency position it can 
be argued that the only appropriate control condition against which to assess excitatory conditioning is a random (zero CS-US contingency) stimulus. On this basis, Rescorla $(1967,1969$ a) suggests that the commonly employed unpaired CS control (which has a negative CS-US contingency) is inappropriate, since such an unpaired stimulus may be expected to acquire inhibitory properties which would inflate any conditioning estimates calculated from such a control.

The present study demonstrated reliable conditioning when a stimulus was positively correlated with the US (CS + US) and found no reliable difference between autonomic performance to a stimulus uncorrelated with the US (zCS group) and a stimulus which was negatively correlated with the US (nCS group). In spite of the lack of discrimination between the zCS and nCS groups on the autonomic measure, the $\mathrm{SC}$ measure very reliably discriminated the CS-US relations of the three groups. The data suggest that in single-cue autonomic conditioning there is no reliable empirical difference (even in a 90 -subject study) between random and unpaired control procedures even when subjects are well aware of the differing CS-US relations of the two control stimuli. This conclusion is consistent with earlier examinations of the double-cue, differential conditioning paradigms with GSR and digital pulse volume change as the dependent autonomic variables (e.g., Furedy, 1974).

\section{REFERENCE NOTE}

1. Dawson, M. E. Human classical conditioning and relational learning: Validation of questionnaires. Paper presented at a symposium, Classical conditioning and cognitive processes, at 11 th Annual Meeting of the Society for Psychophysiological Research, St. Louis, 1971.

\section{REFERENCES}

Baer, P. E., \& Fuhrer, M. J. Cognitive processes during differential trace and delayed conditioning of the GSR. Journal of Experimental Psychology, 1968, 78, 81-88.
BaER, P. E., \& Fuhrer, M. J. Cognitive processes in the differential trace conditioning of electrodermal and vasomotor activity. Journal of Experimental Psychology, 1970, 84, 176-178.

Cronbach, L. J. Essentials of psychological testing. New York: Harper \& Row, 1960.

Dawson, M. E. Cognition and conditioning: Effects of masking the CS-US contingency on human GSR classical conditioning. Journal of Experimental Psychology, 1970, 85, 389-396.

Dawson, M. E., \& Biferno, M. A. Concurrent measurement of awareness and electrodermal classical conditioning. Journal of Experimental Psychology, 1973, 101, 55-62.

Dawson, M. E., \& Grings, W. W. Comparison of classical conditioning and relational learning. Journal of Experimental Psychology, 1968, 76, 227-231.

FUREDY, J. J. Experimental assessments of the importance of controlling for contingency factors in human classical differential electrodermal and plethysmographic conditioning. Psychophysiology, 1974, 11, 308-314.

Furedy, J. J., \& Schiffmann, K. Test of the propriety of the traditional discriminative control procedure in Pavlovian electrodermal and plethysmographic conditioning. Journal of Experimental Psychology, 1971, 91, 161-164.

Morgenson, D. F., \& Martin, I. The orienting response as a predictor of autonomic conditioning. Joumal of Experimental Research in Personality, 1968, 3, 89-98.

Rescorla, R. A. Pavlovian conditioning and its proper control procedures. Psychological Review, 1967, 74, 71-80.

Rescorla, R. A. Pavlovian conditioned inhibition. Psychological Bulletin, 1969, 72, 77-94. (a)

Rescorla, R. A. Conditioned inhibition of fear. In N. J. Mackintosh, \& W. F. Honig (Eds.), Fundamental issues in associative learning. Halifax: Dalhousie University Press, 1969. (b)

Schiffmann, K., \& Furedy, J. J. Failures of contingency and cognitive factors to affect long-interval differential Pavlovian autonomic conditioning. Journal of Experimental Psychology, 1972, 96, 215-218.

SHEAN, G. D. Vasomotor conditioning and awareness. Psychophysiology, 1968, 5, 22-30. (a)

SheAN, G. D. The relationship between ability to verbalize stimulus contingencies and GSR conditioning. Journal of Psychosomatic Research, 1968, 12, 245-249. (b)

(Received for publication June 11, 1976; revision received December 29, 1976.) 